\title{
Die Schwarze Theologie von Allan Boesak - mit besonderem Hinweis auf seine Anthropologie
}

\author{
J H (Amie) van Wyk \\ School for Ecclesiastical Studies \\ Potchefstroom University for CHE
}

\begin{abstract}
$^{1}$
The Black Theology of Allan Boesak - with specific reference to his anthropology

Allan Boesak is a well known theologian and political reformer in the South African context. In this article the author scrutinises the political theology of Boesak in general and his anthropology in particular. The last section of the article is devoted to an assessment of Boesak's contribution as developed within the broader context of the theology of liberation. The investigation clarifies the fact that, although Boesak represents a more moderate kind of liberation theology, serious questions could still be raised about some of his insights.
\end{abstract}

\section{EINLEITUNG}

Es ist bekannt, daß die Schwarze Theologie ihren Ursprung in Amerika hatte (James Cone), jedoch von dort auch Südafrika stark beeinflußte (cf. Kalilombe, 1955:192-216; Pityana, 1994:173-183). Allan Boesak ehemaliger

Konsistorialpräsident der Niederdeutsche Reformierte Missionskirche ${ }^{2}$ und Präsident des Weltbundes Reformierter Kirchen, war einer der ersten und freimütigsten Redeführer dieser Theologie. Boesaks Beteiligung am struggle in Südafrika und seine scharfe Opposition gegen Apartheid machte ihn zu einer

\footnotetext{
${ }^{1}$ A slightly reworked version of a lecture presented at the Faculty of Theology of the University of Rostock, Germany, in 1996. Translation by prof L R Tönsing and dr C E Hentschel, PUCHE.

${ }^{2}$ Nederduits Gereformeerde Sendingkerk - das war damals der Name dieser Kirche.
} 
weltbekannten Figur. Daß Boesak später die Kirche und Theologie für die Politik eingetauscht hat, beeinträchtigt nicht die theologischen Erkenntnisse, die er leidenschaftlich vertreten hat und die nach eigenem Wert beurteilt werden sollten.

Boesaks Theologie wird sehr unterschiedlich beurteilt - ein Aspekt, den wir später noch eingehend behandeln werden. Selbstverständlich fand er bei schwarzen Theologen großen Beifall, während er von weißen Theologen scharf kritisiert wurde. Scholtz (1989:142), der ein Buch über Boesak publizierte, meint, Boesaks Theologie könne "keineswegs reformiert" genannt werden und Smit (1982:16) urteilt, wir hätten es bei Boesak mit einem anderen Evangelium zu tun. Trotzdem fällt es auf, daß Boesak im Rahmen der reformierten Traditionen theologisiert und sich oft auf Calvin beruft (cf. Boesak 1979a:12, 13, 54; 1984b:26, 98, 99, 100-101, 115, 118; 1986b:32).

Um Boesaks Anthropologie, die ein so wichtiges Element seiner Theologie ist, richtig zu verstehen, muß man sie im Zusammenhang mit seinen anderen theologischen Erkenntnissen behandeln. Deswegen widmen wir uns erst diesem breiteren theologischen Kontext, bevor wir uns seiner Anthropologie zuwenden. Ferner müssen wir festhalten, daß Boesaks Theologie nur dann richtig verstanden werden kann, wenn sie in dem breiten soziopolitischen Kontext der Apartheid untersucht wird.

\section{THEOLOGISCHER KONTEXT}

\subsection{Schriftverständnis}

Boesak betrachtet die Schrift als höchste Autorität und Norm (Boesak 1984b:9497). Schriftautorität ist die einzige Norm in der Ethik (Boesak 1977:16; 1979a:13), auch in der Situationsethik (Boesak 1977:86, 112, 113). Bei Boesaks Schriftverständnis sollte in Betracht gezogen werden, daß es eine Schrift ist, die das volle Leben beleuchtet (Boesak 1977:36; 1986a:64) und daher allem (westlichen) dualistischen und verteilenden Denken sowie der Spritualisierung des Evangeliums abgeneigt (Boesak 1977:23, 71, 72, 111) und folglich auf einen 
holistischen Ansatz ausgerichtet ist (Boesak 1977:17). Die zentrale Frage ist, ob Boesak der Gefahr ideologischer Prämissen entkommt (Scholtz 1989:132), eine Gefahr, die er selber signalisiert (Boesak 1977:80). Es ist ja möglich, die Autorität der Schrift vorbehaltlos zu akzeptieren und trotzdem die Schrift für eine bestimmte Ideologie zu mißbrauchen. Boesak achtet neutrale Theologie als unmöglich und meint, daß nur eine Theologie, die das Thema Gott als Befreier der Armen und Unterdrückten ins Zentrum stellt, nicht einer Ideologie verfällt (Boesak 1977:85). Er betrachtet Schwarze Theologie als die theologischen Überlegungen schwarzer Christen über die Situation, in der sie leben, und über ihren Freiheitsstreit (Boesak 1977:9). Theologie ist ein kritisches Nachdenken über historische Praxis (Boesak 1977:16).

\subsection{Gotteslehre}

In der Gotteslehre wiederholen sich die bekannten Themen. Gott ist der Gott des Exodus und der Befreiung (Boesak 1977:15, 20-22, 85; 1984b:8), der Gott der Armen (Boesak 1977:21, 85; 1979a:2; 1984b:9; 1985b:65-73).

\subsection{Sündenlehre}

Scholtz (1989:123) meint, daß Boesak die Sünde "ausschließlich" auf den politischen und sozialen Bereich bezieht und daß er die Sünde "reduziert" auf Apartheid und Kapitalismus (Scholtz, 1989:135). Boesak (1984b:97) ist der Überzeugung, daß er die Zerrissenheit des Lebens gerne eingestehen wolle, aber daß in der Theologie der Apartheid der Fehler begangen wurde, diese Zerrissenheit zu akzeptieren, zu idealisieren und zu "institutionalisieren" - Sünde sollte jedoch bekämpft werden.

\subsection{Christologie}

In der Christologie wird Christus als Befreier betont (Boesak 1977:25; 1984b:13) sowie als Gönner der Armen und Unterdrückten (Boesak 1977:38-39; 1984b:12). Boesak betrachtet Befreiung sogar als das zentrale Thema des Evangeliums (Boesak 1977:18, 19, 34, 107), aber dann handelt es sich um eine totale 
Befreiung (Boesak 1977:14, 17, 27) des ganzen Menschen (Boesak 1986a:61) und nicht um einen Teil des Menschen. Scholtz (1989:121, 122, cf. 135) urteilt also etwas einseitig, wenn er schreibt, daß bei Boesak "keine Rede" von geistlicher Erlösung ist. ${ }^{3}$ Ohne Zweifel legt Boesak einen schweren Akzent auf "politische Befreiung", aber das muß verstanden werden als Reaktion gegen eine einseitige pietistisch-spiritualistische Verengung des Evangeliums in der Apartheidstheologie. Boesak betont auch die Universalherrschaft Christi und den unbedingten christlichen Gehorsam (Boesak 1979a:10, 13; 1984b:103; 1986a:64).

\subsection{Ekklesiologie}

In der Ekklesiologie wird um Beachtung der Kirche der Armen gebeten (Boesak 1977:116; 1985b:68-70). In Südafrika haben wir nach Boesak die tragische Lage, daß die reformatorischen Kirchen Rassismus rechtfertigten. "Rassismus ist die unvermeidliche Frucht der reformierten Tradition“ (Boesak 1984b:93). ${ }^{4}$ Vor allem muß die Nederduitse Gereformeerde Kerk sich immer wieder Boesaks scharfe Kritik gefallen lassen (Boesak 1979b:147-158). ${ }^{5}$ Die (weiße) kirchliche Situation ist so bedenklich, daß man sagen muß, die Kirche sei ein Wolf geworden (Boesak 1979a:55). Begreiflich ist daher sein Plädoyer für eine eigene und neue Konfession (Boesak 1984b:103); mit "Beichte von Belhar"6 ist dieser Traum sicher in Erfüllung gegangen. ${ }^{7}$

Boesak betrachtet Wortverkündigung als Hauptaufgabe der Kirche (1979a:6). Die prophetische Berufung der Kirche in der Geselschaft kommt unter

\footnotetext{
3 Auch Smit (1988:52) ist der Meinung, daß ein "himmelsbreiter Unterschied" zwischen Boesaks Befreiungstheologie und reformierter Erlösungstheologie ist.

4 "Racism is an inevitable fruit of the reformed tradition" (Boesak 1984b:93).

${ }^{5}$ Scharfe Kritik wird auch gegen die theologische Begründung der Apartheid durch E P Groenewald geäußert (Boesak 1977:32-33).

${ }^{6}$ Belydenis van Belhar.

7 Gegen dieses Kolorit muß seine Unterzeichnung von The Road to Damascus (1989) gesehen werden. Verwunderlich ist jedoch daß er The Kairos Document (1985) nicht unterschrieben hat.
} 
die Lupe. Statt einseitiger pietistischer Predigung (Boesak 1977:23; 1979a:4, 7), will er relevante politische Predigung (Boesak 1977:116; 1979:10, 15). Das Evangelium (und die Kirche) darf nicht verideologisiert werden (Boesak 1986a:66) und darf nicht mit einer Kultur, einem politischen Programm, einer sozialen Ideologie oder einem moralischen System vereint werden (Boesak 1977:79, 95, 96) - es muß immer eine kritische Distanz gewahrt werden - aber dennoch darf man nicht zögern, die Herrschaft Christi über das ganze Leben zu verkündigen (Boesak 1979a:10,11). ${ }^{8}$

Boesak liefert selber eine lebende Illustration dieser prophetischen Predigung durch die Publikation einiger Predigtbände. ${ }^{9}$ Er predigt über die Beendigung (den Fall) einer ungerechten Regierung (Boesak 1986b:30-40; cf. 1985b:70-71) - die südafrikanische Regierung wird als das Tier aus dem Meer gesehen (cf. Offenbarung 13) (Boesak 1986b:151; 1987:101). ${ }^{10}$

Für Boesak ist die Kirche nicht Selbstzweck, sondern Instrument zu einem Ziel, nämlich das Königreich (Boesak 1986a:65). Das Königreich überschreitet Grenzen und bringt den Armen Gerecthtigkeit (Boesak 1984b:109), ist jedoch mehr als (sogar eine gerechte) politische Einrichtung (Boesak 1979a:16); auch Schwarze Macht ist nicht imstande, das Königreich Gottes realisieren zu lassen (Boesak 1977:79). Wohl ist der Mensch imstande, Zeichen des Königreiches sichtbar zu machen (Boesak 1986a:63). Schließlich mündet alles aus in einen neuen Himmel und eine neue Erde (Boesak 1987:126-138), welches eine Erneuerung dieser Erde beinhaltet (Boesak 1987:129).

\section{ANTHROPOLOGIE}

Gegen diesen Hintergrund müssen Boesaks Ansichten des Menschen gesehen werden. Ohne Übertreibung kann behauptet werden, daß Boesaks

\footnotetext{
${ }^{8}$ Nicol (1986:34) ist der Meinung, daß Scholtz Boesak hier falsch versteht.

${ }^{9}$ Cf Die vinger van God (1979a), Walking on Thorns (1984a) und Machtigen heeft Hij van de troon gestoten (1989).

10 Während K. Runia im Centraal Weekblad (1981-03-11) der Meinung ist, daß die Predigten in Die vinger van God "biblisch verantwortet und eindringend" sind, urteilt H De Waarde (1985:5455) über Walking on Thorns: "Boesak ist in Gefahr, das Evangelium zu einer Botschaft politischer und sozialer Befreiung” zu reduzieren.
} 
leidenschaftliche Auflehnung gegen Apartheid, seine Verwerfung dieser Ideologie (und dazu auch der christlich-nationalen Gedankens (Boesak 1977:92, 94, 96) seine Anthropologie wesentlich bestimmt. Apartheid verursacht ja, daß Menschlichkeit durch die weiße Hautfarbe bestimmt wird (Boesak 1979a:43); Apartheid bringt mit sich eine totale Kontrolle und Determinierung des Lebens der Schwarzen aufgrund ihrer Hautfarbe (Boesak 1984b:6). Es ist "ein falscher Gott, dessen autoritäre Unverschämtheit dem Wesentlichen einer sinnvollen Humanität keinen Raum läßt: Freiheit unter Gott” (Boesak 1984b:105). ${ }^{11}$ Apartheid dehumanisiert (Boesak 1984b:2), denn sie definiert Humanität nach weiß und nicht-weiß (Boesak 1984b:4); daher: "Das Christentum in Südafrika ist geweißt" (Boesak 1977:92). ${ }^{12}$ "Aufgrund dieser Sich wird Apartheid zurückgewiesen in sehr ausgesprochener Terminologie. Apartheid ist Sünde (1979a:43), ein Pseudo-Evangelium (1982:285-288; 1984b:52, 93, 110), ein Abgott (Boesak 1979a:70): ${ }^{13}$

Es ist wichtig, Apartheid als unversöhnlich mit dem Evangelium zu bezeichnen, eine Sünde, die auf jeder Lebensebene bekämpft werden muß, eine Leugnung der Reformierten Tradition, ein Irrglaube - das ist zur ewigen Schande der Kirche Jesu Christi in der Welt

(Boesak 1984b:103)

Apartheid ist Rassismus und Systemzwang (Boesak 1984b:48), strukturierte Sünde (Boesak 1984b:110f), eine Ideologie von Rassendominanz und eine Negierung der Eigenart anderer; das heißt, das Wichtigste an einer Person ist nicht, daß sie nach dem Bild Gottes mit unveräußerlichen Rechten geschaffen ist, sondern wohl ihre Rassenidentität (Boesak 1984b:114). Für einen Schwarzen bedeutet Apartheid "primitive Wohnungen, unterbezahlt,

11 It is "a false God, whose authoritarian audacity allows no room for the essence of meaningful humanity: freedom under God".

12 "Christianity is whitenized in South Africa."

13 "It is important to declare apartheid to be irreconcilable with the gospel of Jesus Christ, a sin that has to be combatted on every level of our lives, a denial of the reformed tradition, a heresy that is to the everlasting shame of the church of Jesus Christ in the world." 
Ausweiskontrolle, Zustrombeschränkungen, Wanderarbeiter, "group areas", Umsiedlungslager, Ungleichheit vor dem Gesetz, Furcht, Einschüchterung, weiße Vorgesetzte und schwarze Spitzel, Herablassung und Paternalismus" (Boesak 1977:49). ${ }^{14}$ Südafrika wird als das meist rassistische Land der Welt gesehen (Boesak 1984b:109) mit dem homeland-Programm als größte Immoralität des Rassismus (Boesak 1984b:51).

Apartheid ist vollkommen im Gegensatz zur Humanität Christi, dem wir nachfolgen sollten (Boesak 1984b:11); Apartheid ist auch im Gegensatz zur Liebe und Gerechtigkeit, durch die die Menschheit ihre volle Humanität realisiert (Boesak 1984b:11). Versöhnung (in der Politik) ist nur möglich, nachdem (nicht: durch) (soziale) Gerechtigkeit wieder hergestellt ist (Boesak 1977:75).

Nach Boesak muß Apartheid bekämpft werden; es handelt sich um einen Befreiungs- und Dekolonisierungskampf (Boesak 1984b:6). Nicht die Weißen sollen gehaßt werden, wohl aber die weiße Unterdrückung (Boesak 1977:108; 1984b:18). "Schwarze wissen ganz genau, daß jeder Weiße, solange er Unterdrücker ist, kein Bruder sein kann. Er ist ein Feind" (Boesak 1977:28). ${ }^{15}$ Schwarze müssen ihre eigene Humanität entdecken.

Unsere wahre Humanität besteht gerade darin, daß wir als Schwarze geschaffen sind. Es wird uns nie gelingen, bessere Beziehungen zu Weißen zu haben, bis die Weißen nicht gelernt haben, Schwarze als schwarz Menschen zu akzeptieren

(Boesak 1984b:16) $^{16}$

Schwarzes Bewußtsein bedeutet, daß Schwarze einsehen, daß Annahme ihrer schwarzen Hautfarbe notwendig ist für ihre Humanität (Boesak 1977:26-27, 110).

\footnotetext{
${ }^{14}$ For blacks apartheid meant "bad housing, being underpaid, pass laws, influx-control, migrant labour, group areas, resettlement camps, unequality before the law, fear, intimidation, white bosses and black informers, condescension and paternalism."

15 "Blacks know with realistic clarity that any white person, as long as he is an oppressor, can never be a brother. He is an enemy."

16 "Our true humanity consists precisely in our creation as Blacks. We shall never be able to gain better human relations until whites have learned to accept Blacks as Black people ...".
} 
Schwarze müssen von einem falschen Selbstbild, daß schwarz minderwertig sei, und einer falschen Ideologie, daß weiß mehrwertig sei, befreit werden; und Weiße müssen von ihrer Angst und ihrem Unrecht und ihrer Herrschaft befreit werden (cf. Kirk, 1980:108-113). Menschen sind nach dem Bild des mächtigen Gottes geschaffen. Hieraus folgt, daß des Mensch auch über Macht verfügt, aber dann über Macht, die befreit, die dient, die Recht und Gerechtigkeit schafft ansonsten ist Macht unmenschliche, teuflische und selbstvernichtende Macht (Boesak 1977:44, 45, 48). Nicht Integration ist für Schwarze wichtig, sondern Befreiung (Boesak 1977:107) - und Heilsein (Boesak 1984b:53).

In der Politik geht es um mehr als das Mögliche (Boesak 1986a:63); es geht ja doch um Menschen (Boesak 1986a:63), und deswegen muß ein unterdrückendes System, das Menschen entmenschlicht, radikal geändert werden. Interessanterweise warnt Boesak nicht nur gegen Verknechtung durch Kapitalismus, sondern auch durch Marxismus (Boesak 1987:131132). Die große Frage ist jedoch: muß die Änderung mit oder ohne Gewalt geschehen? Wiederstand muß sein - Apartisten werden ja nie grundsätzlich Änderung unterstützen (Boesak 1986a:65) - sie sind ja zu ängstlich (Boesak 1979a:53), aber darf sie dann gewaltsam sein?

Diese Transformation der Gesellschaft könnte eine Revolution genannt werden, aber sie muß nicht notwendigerweise gewalttätig sein. Wir verstehen eine solche Revolution als einen fundamentalen sozialen Wandel" (Boesak 1977:114).

Wir wollen nicht leugnen, daß eine Situation entstehen könnte, wo den Unterdrückten keine andere Wahl mehr bleibt als die Gewalt der Vergeltung; dabei zögern wir jedoch, denn wir wissen, daß dies sich wahrscheinlich als schlechte 'Lösung' herausstellen wird und daß Gewalt nie 'gerechtfertigt' werden kann. (Boesak 1977:58) ${ }^{17}$

\footnotetext{
17 "This transformation of society can be called a revolution and this revolution need not necessarily be violent. We understand such a revolution to be a fundamental social change."

"(W)e do not deny that a situation may arise where retaliatory violence is forced upon the oppressed and no other avenue is left open to them, [but] we do so with a clear hesitancy, knowing full well that it will probably prove a poor 'solution' and that violence can never be 'justified'."
} 
Über Auflehnung können nur Schwarze (als Unterdrückte), nicht Weiße (als Unterdrücker) beschließen (Boesak 1977:56, 57, 100). Boesak hat lange für gewaltlosen Widerstand gekämpft - Gewalt bietet seines Erachtens keine Lösung (Boesak 1986a:16-20; 1986b:35). Er hatte jedoch Verständnis für Gewalt (Boesak 1986a:20). Später, als er merkte, daß gewaltlose Aktionen keine Resultate aufweisen, wurde seine Haltung ambivalenter (Boesak 1989:101-106).

Mit Recht urteilte er, daß Weiße heuchlerisch und unehrlich waren, indem sie ohne Motivierung Schwarze Gewalt verurteilten, während die reformatorische Ethik noch immer Gewalt in der Form eines gerechten Krieges oder einer gerechten Rebellion akzeptiert hat (cf. Calvin). Aussprachen, daß keine Form der Gewalt für einen Christen akzeptabel ist (Scholtz, 1989:136) und daß jegliche Form gewaltsamen Widerstandes gegen eine Regierung unakzeptabel ist (Scholtz, 1989:144), sind gut gemeint, waren aber noch nie Teil der reformierten Tradition - wohl der pazifistischen. ${ }^{18}$ In der reformierten Theologie stand eine Wahl zwischen Gewalt und keiner Gewalt nie zur Debatte, wohl aber die zwischen rechtmäßiger und unrechtmäßiger Gewalt. Wer die Schwarze Gewalt verurteilt, muß das tun, weil sie unrechtmäßige Gewalt is, ein Argument, welches dann selbstredend motiviert werden muß.

\section{WERTBESTIMMUNG}

Eine fundierte Evaluierung muß zweierlei hervorheben: einerseits die Herausforderungen der Schwarze Theologie bzw. Anthropologie an die herkömmliche Theologie, andererseits die damit verbundenen Gefahren (cf. Gutiérrez 1974; Boff \& Boff 1989; Brown, 1990; Villa-Vicencio 1994:184-196; Sobrino \& Ellacuría 1996; Maimela 1998:11-119.)

Betreffs der Herausforderung wird auf fünf Aspekte hingewiesen.

${ }^{18}$ Der Pazifismus führt diese Argumente, die schwerwiegend sind - Argumente, die meines Erachtens theologisch viel stärker sind als die Theologie der Revolution - aber das gehört nicht hierhin (cf. Van Wyk 1974). 


\subsection{Herausforderungen}

\subsubsection{Der ganze Mensch}

Erstens wird unsere Aufmerksamkeit auf den Menschen, den konkreten, irdischen, vollen Menschen gerichtet. Unserer Aufmerksamkeit wird nicht nur auf die Seele wie im Pietismus, nicht nur auf den Weißen, wie in dem Apartismus, nicht nur auf Männer wie im Androzentrismus gelenkt, sondern auf den Menschen als Mensch, auf jeden Menschen. Knechten und Entrechten, Unterdrückung und Entmenschlichung wird der Kampf angesagt zugunsten einer totalen und radikalen Befreiung des Menschen.

\subsubsection{Der strukturierte Mensch}

Zweitens handelt es sich um den strukturierten Menschen, den Menschen innerhalb Strukturen. Man hat entdect, daß eine einseitige, individualistische Menschensicht nicht ausreicht, da das Leben des Menschen von A bis $Z$ durch politische und wirtschaftliche Strukturen und Systeme mitbestimmt wird. Das Sein bestimmt das Bewußtsein, das System den Menschen. Deswegen muß im Falle der Knechtung und Versklavung nicht nur das Herz des Menschen sich ändern, sondern auch die politischen und wirtschaftlichen Strukturen. ${ }^{19}$

Auch politische Strukturen wie Minderheitsregierungen wurden in Frage gestellt und er wurde zugunsten einer demokratischen Regierungsform argumentiert - eine Angelegenheit, die gehandhabt werden muß, nachdem eine Revolution vollzogen ist (cf. Maimela 1990:198). So sträubte Boesak sich zum Beispiel gegen die südafrikanische Regierung als eine ungerechte/illegitime Regierung. ${ }^{20}$

\footnotetext{
19 Wird Versklavung und Knechtung aufgehoben, wird stets der Sozialismus vorgezogen und dem Kapitalismus kritisch begegnet, obwohl diese Haltung heutzutage wegen der Zerbröckelung des Sozialismus in Ost-Europa (1989) und der Sowjetunion (1991) unter gewaltigen Druck gekommen ist.

20 In diesem Zusammenhang müssen wir meines Erachtens unterscheiden zwischen der Frage, wer regiert (die Frage nach der Legalität/RechtmäBigkeit) und der Frage, wie regiert wird (die Frage nach der Legitimität/Gerechtigkeit). Calvin (Inst. 4.20.8) hat uns gelehrt, daß die wichtigste Frage nicht ist, wer regiert (Aristokratie, Oligarchie, Demokratie), sondern wie regiert wird. In einer gebrochenen Wirklichkeit ist es ja möglich, daß eine unrechtmäßige Obrigkeit (eine Autokratie oder Minderheitsregierung) gerecht (oder ungerecht) riegieren kann und eine rechtmäßige Obrigkeit (Demokratie) ungerecht (oder gerecht). Afrika z.Z. wird gekennzeichnet durch viele illegale Regierungen, aber das heißt nicht, daß sie notwendig illegitim sind. Selbstverständlich wird die meist ideale Situation dort angetroffen, wo die wer- und die wie-Frage engstens verbunden sind und eine rechtmäßige Obrigkeit (plurale Demokratie) gerecht regiert.
} 


\subsubsection{Der protestierende Mensch}

Drittens wenden wir uns dem protestierenden Menschen zu. In der revolutionären Menschensicht gibt er keinen Raum für gelassene Akzeptierung, für menschliche Erniedrigung, für blinden Gehorsam und für strukturierte Gewalt. Die allermeisten Menschen auf Erden - die Armen und die Frauen - sind Unterdrückte und Verunrechtete und es ist ihre Berufung, gegen Diskriminierung und Dominierung, gegen Rassismus und Paternalismus zu protestieren und die Fesseln der Knechtung zu sprengen. Nicht Gehorsam, sondern Ungehorsam (zumindest kein blinder Gehorsam) soll die christliche Lebenshaltung tipieren. Es kann nicht die Rede sein von einem lojalen und echten Gehorsam an eine Regierung, die ungerecht regiert. Gegen eine solche kann man nur protestieren, sogar rebellieren, ja revolutionieren. Tatsächlich ist die reformatorische Tradition nicht pazifistisch, aber sie hat stets jegliche Auflehnung gegen eine Obrigkeit strengen Bedingungen unterworfen, während die revolutionierende Menschensicht hier die größte Freiheit zugelassen hat.

\subsubsection{Der prophezeiende Mensch}

Viertens wollen wir den prophezeienden Menschen betrachten, d.h. die prophetische Berufung des Christen und der Kirche in einem soziopolitischen System von Unrecht und Unterdrückung. Der Gedanke, daß ein Christ oder seine Kirche nicht politisch engagiert sein können, ist immerhin eine Illusion (cf. Maimela 1990:166). Negativität und Neutralität hier wären nichts anderes als Unterstützung des status quo. Der Gläubige hat eine verpflichtenden prophetische Berufung und darf sich nicht in einer pietistischen Lebenshaltung zu einer passiven Haltung des Stillschweigens (Quietismus) bewegen lassen (cf. Vorster, 1984:124-126). Obwohl die Kirche auf der Hut sein sollte gegen die Gefahr ihrer Verpolitisierung, müssen wir zugeben, daß dieser Aspekt der kirchlichen Verantwortung in der Gesellschaft in der reformatorischen Theologie zu lange vergessen und unterentwickelt war (cf. Van Wyk 1991:98-104; 205212). 


\subsubsection{Der benachteiligte Mensch}

Fünftens wird dem benachteiligten Menschen, dem Armen, dem Unterdrückten, der Randfigur besonderes Interesse gewidmet. Nicht nur die Reichen, sondern auch die Armen, nicht nur die Weißen, sondern auch die Schwarzen, nicht nur die Männer, sondern auch die Frauen kommen zu ihrem Recht. Man könnte in diesem Zusammenhang fast von einer Theologie der Armen reden (ein Aspekt, über den schon viele Bücher und Artikel erschienen sind.)

Es ist klar, daß Armut eine weltweite Erscheinung ist und also auch internationale Aufmerksamkeit verdient. Aus der Geschichte geht hervor, daß Afrika ein Kontinent ist, das in großem Ausmaß von Kolonialmächten ausgebeutet wurde und deshalb auf Kompensation berechtigt ist. Aus der Bibel geht klar hervor, daß Reiche eine besondere Verantworung für die Armen haben (cf. nur die Propheten und Lukas). Andrerseits dürfen die Armen auch nicht in ihrer Armut resignieren, sondern benötigen einen Arbeitsethos ohne Arbeitsscheu und einseitige Kontemplation, wie dies in Afrika so häufig vorkommt.

Dann muß auch auf einige Gefahren der Schwarzen Theologie/Anthropologie hingewiesen werden:

\subsection{Gefahren}

\subsubsection{Eine optimistische Menschensicht}

Erstens muß auf die Gefahr einer optimistischen Menschensicht mit ihrer notwendigen Kehrseite einer minimalisierenden Sündenauffassung hingewiesen werden (cf. Heyns 1975:71; Vorster 1984:139-143; Potgieter 1988:74-76). In einer optimistischen Menschensicht, in der der Mensch selber für seine Erlösung verantwortlich ist, oder zumindest daran mitwirkt, und der Mensch imstande ist, das Reich Gottes eigenhändig zu verwirklichen, wird das zerstörende Werk der Sünde gänzlich unterschätzt und das menschlichte Vermögen total überschätzt. Pelagianismus und Calvinismus sind nun einmal nicht miteinander zu versöhnen. 
Diese Neigung kommt bei anderen Exponenten der Schwarzen Theologie häufiger als bei Boesak vor.

\subsubsection{Eine gesetzliche Menschensicht}

Eng hiermit verbunden ist eine gesetzliche Menschensicht. Das Evangelium erzählt von einer Erlösung, die durch Christus vollbracht worden ist, während nach der revolutionären Menschensicht die Erlösung unvollendet ist und (durch den Menschen) vollbracht werden muß. Bestimmte Formen der Befreiungstheologie bewegen sich weg von dem Evangelium der befreienden Gnade hin zum Gesetzeszwang und sind dadurch eine "Vergesetzlichung des Evangeliums: und folglich eine Verzerrung, ja sogar Abtötung desselben" (Engelbrecht 1978:50-51). Mit Recht beschwerte die revolutionäre Menschensicht sich gegen die Reduzierung der Erlösung zu nur einer Dimension, aber leider wird die Unterscheidung zwischen Heil und Heiligung herdurch nur zu leicht preisgegeben. Wentsel (1991:656) spricht in diesem Zusammenhang von einem "mehrdimensionalen Heilsbegriff" und unterscheidet zwischen Befreiung und Erlösung (Wentsel 1991:651-658). Nichts ist weiter von der Gnade entfernt als der Versuch des Menschen, sich selber zu retten.

\subsubsection{Eine verpolitisierte Menschensicht}

Drittens droht die Gefahr einer verpolitisierten Menschensicht (cf. Heyns 1975:51; Engelbrecht 1978:12-39; Vorster 1984:157-163). In dieser Menschensicht wird fast alles in politische Terminologie übersetzt: Gott/Christus ist ein politischer Befreier, Sünde ist politische Sünde, Heil ist politisches Heil, die Kirche ist politischer Agitator, Glaube ist politische Praxis, das Reich Gottes ist eine politische Utopie, der Mensch ist homo politicus. Zugegeben, daß die Politik - und Wirtschaft - einen großen Einfluß auf das Leben des Menschen ausüben, bleibt das doch nur ein Aspekt des Lebens und nicht das ganze Leben. Das menschliche Leben darf nicht zu Politik reduziert werden; der Mensch ist mehr als Politik. Alles ist Politik, aber Politik ist nicht alles, hat Kuitert (1985) mit Recht gesagt. 


\subsubsection{Eine gewalttätige Menschensicht}

Viertens muß die Frage in bezug auf die revolutionäre Menschensicht an sich gestellt werden. Es ist wahr, daß der Mensch nicht mit dem Bösen und Unmenschlichen in der Gesellschaft mitmachen darf, aber muß sein Protest notwendig gewalttätig sein? Ist das Christentum per Definition revolutionär und ist antirevolutionäres Christentum kein Christentum? Befreit "befreiende Gewalt" tatsächlich, oder nimmt sie nur wieder neue Formen von unterdrückender Gewalt an? Die reformatorische Menschensicht kann alles andere als revolutionär betrachtet werden - dafür ist das menschliche Leben und die menschliche Person zu hoch geschätzt. Mit dieser Aussage ist jedoch kein Paß für "strukturelle Gewalt" gegeben, sondern wird gesagt, daß die Normen, nach denen, das Ziel, wozu, die Methode, nach der und die Mittel, mit denen die Transformation der Gesellschaft unternommen wird, nicht Moralität und Verantwortung negieren dürfen. Ist Rassismus eine Form des menschlichen Hasses, ist Revolutionismus es nicht auch? Ist die theologische Rechtfertigung der Apartheid Ketzerei, ist die theologische Rechtfertigung einer Revolution es nicht auch?

\subsubsection{Eine utopistische Menschensicht}

Fünftens kann die Gefahr einer utopistischen Menschensicht nicht umgangen werden (cf. Heyns 1975:53-54; Engelbrecht 1978:56; Vorster 1984:136-139). Da die revolutionäre Menschensicht den "eschatologischen Vorbehalt" verkennt, sieht sie den Menschen als verwirklicher des Reiches Gottes. Tatsächlich ist das Köningreich (schon) gegenwärtig, aber es ist (in seiner Finalität) auch zukünftig, es ist irdisch, aber auch himmlisch, es ist offenbar, aber auch verborgen, es ist Auftrag an den Menschen, aber schließlich Gabe Gottes. In der revolutionären Menschensicht wird Gott schlußendlich von menschlichen Taten abhängig gemacht und ändert die eschatologische Erwartung sich in einen utopischen Traum. Gottes Königreich ist immer größer als unsere menschlichen Königreichlein und seine Taten stets dauerhafter als die unsrigen. Auch hier rächt das optimistische Menschenbild sich. 
Das Königreich Gottes ist eine umfassende Angelegenheit. Es umfaßt Geist und Körper, Mensch und Natur, Erde und Himmel. Das Königreich läßt sich nicht verinnerlichen noch veräußerlichen, verirdischen noch verhimmlischen. Auch umschließt das Königreich mehr als die Befreiung des Menschen von politischen Strukturen, selbst mehr als die Erlösung des Menschen aus Sünde, ja es schließt endlich die totale Erneuerung der ganzen Schöpfung ein. Weswegen hören wir in der revolutionären Menschensicht so viel von dem unterdrückten Menschen und so weinig von der unterdrückten Natur, von der Vernichtung von Bäumen und Wäldern, der Ausrottung von Tieren, der Verschmutzung der Umgebung ? Ist Afrika hier etwa der Welt ein Beispiel? Was hülfe es dem Menschen, wenn er den politischen Kampf gewönne, den ökologischen aber verlöre (cf. De Gruchy1991:115)?

\section{SCHLUß}

Zweifels ohne hat Boesak einen besonderen Beitrag zum Ausbau der kontextuellen reformierten Theologie in Südafrika geliefert und die Augen für wichtige Aspekte der Theologie geöffnet. Dennoch kann gegen (verschiedene) seiner theologischen Einsichten Kritik eingebracht werden und konnte er die Versuchung bestimmter Einseitigkeiten nicht überwinden.

\section{Literatur}

Boesak A 1977. Farewell to innocence: A social-ethical study of black theology and black power. Johannesburg: Ravan.

Boesak A 1979a. Die vinger van God: Preke oor geloof en die politiek. Johannesburg: Ravan.

Boesak A 1979b. Tensy ' $n$ wonder gebeur... Die kerkeberaad en die verhouding tussen die wit en die swart NG Kerke, in Meiring, P \& Lederle, H I, (reds), Die eenheid van die kerk, 147-158. Tafelberg: Kaapstad.

Boesak A 1982. The heresy of Apartheid, in Serfontein, J H P (ed), Apartheid, change and the NG Kerk, 285-288. Emmarentia: Taurus.

Boesak A 1984a. Walking on thorns: The call to Christian obedience. Geneva: WCC. 
Boesak A 1984b. Black and reformed: Apartheid, liberation and the Calvinistic tradition. Johannesburg: Skotaville.

Boesak A 1985. God van de armen, in Berkhof, $\mathrm{H}$ et al, Met de moed der hoop: Opstellen aangeboden aan dr C F Beyers Naudé. Baarn: Ten Have.

Boesak A. 1986a. Als dit verraad is ben ik schuldig. Baarn: Ten Have.

Boesak A 1987. Comfort and protest: Reflections on the Apocalypse of John of Patmos. Edinburgh: The Andrew Press.

Boesak A 1989. Machtigen heeft Hij van de troon gestoten: Preken. Baarn: Ten Have.

Boesak A \& Villa-Vicencio, C (ed), 1986. A call for an end to unjust rule. Edinburgh: The Saint Andrew Press.

Boff, L \& Boff, C 1989. Introducing liberation theology. Maryknoll NY: Orbis.

Brown, R M 1990. Gustavo Gutiérrez: An introduction to liberation theology. Maryknoll NY: Orbis.

Calvyn, J 1984/1986/1988/1992. Institusie van die Christelike godsdiens 1559 (1-4).

Potchefstroom: CJBF. (Übersetzer H W Simpson.)

De Waard, H 1985. Rezension. Vox Reformata Nov., 54-55. Nov.

Engelbrecht, B 1978. God en die politiek. Durban: Butterworth.

Gutiérrez, G. 1974. Theologie van de bevrijding. Baarn: Ten Have.

Heyns, J A 1975. Teologie van die revolusie. Kaapstad: Tafelberg.

Kalilombe, P A 1995. Black theology, in Ford, D F (ed), The modern theologians: An introduction to Christian theology in the twentieth century, II, 193-216. Oxford: Blackwell.

Kirk, J A 1980. Theology encounters revolution. Leicester: Inter-Varsity Press.

Kuitert, H M 1985. Alles is politiek maar politiek is niet alles: Een theologisch perspectief op geloof en politiek. Baarn: Ten Have.

Maimela, S S 1990. The emergence on the church's prophetic ministry: an essay on modern trends in theology. Braamfontein: Skotaville.

Maimela, S S 1998. Black theology, in Mailmela, S \& König, A (eds), Initiation to theology: The rich variety of theology and hermeneutics, 111-119. Pretoria: Van Schaik.

Nicol, W 1986. The churches in Southern Africa and the poor - an urgent task. Orientation. (International circular of the PU for CHE), 42:1-40, Sept. 
Pityana, B 1994. Black theology, in De Grucy, J \& Villa-Vicencio, C (eds), Doing theology in context: South African perspectives, 173-183. Cape Town: David Philip.

Potgieter, P C \& Smit, J H (reds) 1988. Vernuwing of konfrontasie. Kaapstad: Lux Verbi.

Scholtz, A 1989. Allan Boesak: Religion und Gewalt. Herford: Busse Seewald.

Smit, J H 1982. Swart teologie, wit teologie en hermeneutiese beginsels. Bloemfontein: UOVS.

Sobrino, J \& Ellacuría, I (eds) 1996. Systematic theology: Perspectives from liberation theology. Maryknoll NY: Orbis.

Villa-Vicencio, H 1994. Liberation theology, in De Gruchy, J \& Villa-Vicencio, C (eds), Doing theology in context: South African perspectives, 184-196. Cape Town: David Philip.

Van Wyk, J H 1974. Etiek van vrede: 'n Teologies-etiese evaluering van die Christenpasifisme. Potchefstroom. (1984. Stellenbosch: Cabo.)

Van Wyk, J H 1991. Moraliteit en verantwoordelikheid: Opstelle oor politieke etiek. Potchefstroom: PU vir CHO.

Vorster, J M 1984. Die Neo-Marxistiese Politieke Teologie in Suid-Afrika: 'n Gereformeerd-apologetiese studie. Potchefstroom: PUCHO. 